\title{
Imaging Profile of the COVID-19 Infection: A Narrative Overview
}

\author{
Mohamed Amine Baba ${ }^{1,2^{*}}$, Ahmed Kharbach ${ }^{1,3}$, Abderrahmane Achbani ${ }^{1,4}$, Aziz Naciri ${ }^{5}$, \\ Youssef Bouchriti ${ }^{1,6}$, Hasna Sine ${ }^{1,7}$
}

\footnotetext{
${ }^{1}$ High Institute of Nursing

Professions and Technical Health,

Agadir, Morocco

${ }^{2}$ Laboratory of Health Sciences, National School of Applied Sciences, Ibn Zohr University, Agadir, Morocco

${ }^{3}$ Laboratory of Biostatistics, Clinical Research and Epidemiology (LBRCE), Faculty of Medicine and Pharmacy of Rabat, MOHAMED V University of Rabat, Morocco

${ }^{4}$ Laboratory of Cell Biology and Molecular Genetics department of Biology, Faculty of Sciences, Ibn Zohr University, Agadir, Morocco

${ }^{5}$ High Institute of Nursing Professions and Technical Health, Laâyoune, Morocco

${ }^{6}$ Faculty of Sciences, Ibn Zohr University, Agadir, Morocco

${ }^{7}$ Medical Biology, Human and experimental pathology and environment, Faculty of Medicine and Pharmacy of Rabat, MOHAMED V University of Rabat, 10100, Morocco
}

\section{Correspondence:}

Mohamed Amine Baba

Address: High Institute of Nursing Professions and Technical Health and Laboratory of Health Sciences, National School of Applied Sciences, Ibn Zohr University Agadir, Morocco

Email:

Mohamedamine.baba@edu.uiz.ac.ma

Received: 28.04.2020,

Accepted: 31.05 .2020

https://doi.org/10.29333/jcei/8319

\section{INTRODUCTION}

An epidemic of Coronavirus Disease 2019 (SARS-CoV-2) began in December 2019 in China leading to a Public Health Emergency of International Concern (PHEIC).

This virus can be transmitted through the respiratory tract and through contact and shows signs of transmission between humans. other possible routes of transmission, faecal-oral and sewage transmission have not yet been confirmed [1-3]. Infection with SARS-CoV-2 is associated with significant morbidity especially in patients with chronic medical conditions. At least one-fifth of cases require supportive care in medical intensive care units, which is especially limited in most developing countries [4].

COVID-19 pneumonia is the most common clinical presentation of SARSCoV-2. The findings on Computed Tomography CT images may reflect the severity of disease. From the start of the pandemic, several studies have emphasized the importance of imaging in the diagnosis and management of pneumonia caused by coronavirus. In the initial screening, an examination by computed tomography (CT) is necessary for the auxiliary diagnosis, the diagnosis is then confirmed by the positive results of the RT-PCR (reverse transcription-amplification in polymerase chain) [5]. CT is considered the first-line imaging modality in highly suspected cases and is helpful for monitoring imaging changes during treatment. Several Chinese researchers strongly recommend CT imaging as the main basis for an early diagnosis of COVID-19 [6].

Thoracic radiology evaluation is often key to the evaluation of patients suspected of COVID-19 infection. Prompt recognition of disease is invaluable to ensure timely treatment, and from a public health perspective, rapid patient isolation is crucial for containment of this communicable disease. Imaging will also make it possible to make a differential diagnosis for patients vulnerable to this pandemic such as patients attacked by lung cancer [7].

Initial discussions after the virus appeared suggested that CT should be the preferred method for the diagnosis of SARSCoV-2 and Chest radiographs are of little diagnostic value in early stages [8]. However, its use for the diagnosis of COVID-19 is controversial [5] other studies recommend a chest ultrasound [9]. Indeed, the place of imaging and radiology staff appears very important in order to fight against this pandemic. 


\section{Sensitivity of Chest CT for COVID-19: Comparison to RT-PCR}

An academician of the American Society for Radiation Oncology called for the immediate establishment of a CTbased diagnostic method for COVID-19 and improvement of the detection rate of the severe acute respiratory syndrome coronavirus 2 (SARS-CoV-2) [1]. If a patient with clinically suspected COVID-19 has negative RT-PCR results but positive imaging results, the patient should be isolated and treated as soon as possible [10].

In a series of 51 patients with chest CT and RT-PCR assay performed within 3 days, the sensitivity of CT for COVID19 infection was $98 \%$ compared to RT-PCR sensitivity of $71 \%(\mathrm{p}<.001)[11]$. The reasons for the low efficiency of viral nucleic acid detection may include: 1) immature development of nucleic acid detection technology; 2) variation in detection rate from different manufacturers;3)low patient viral load; or 4) unsuccessful sampling [11]. Another study assessed lung abnormalities related to disease progression and found that chest CT showed the most extensive disease approximately ten days after the onset of symptoms [12]. On the other hand, and according to the recommendations of the French Radiology Society, there is no indication to carry out a thoracic scanner for the purpose of screening in patients without signs of severity and without co-morbidities, always according to this learned society, the realization of a chest scanner without injection in fine sections is currently indicated in patients with a suspected or confirmed diagnosis and signs of clinical severity (dyspnea, desaturation ...) initial or secondary relating to hospital care. It can also be conceived in suspect patients with co morbidities, awaiting the results of PCR, or else on the first line if the time and availability of PCR become limiting, which seems to be looming [13].

\section{Imaging Profile of the COVID-19 Infection}

The most common findings on chest CT were bilateral ground-glass opacities with or without consolidation in the lung periphery, in a study carried out on a series of 21 cases in Shenzhen and Hong Kong, Pleural effusions and lymphadenopathy were absent, in all patients [14].

A recent Sino-American study showed the possibility of distinguishing SARS-CoV-2 infection from other viral pneumonias, on the basis of the peripheral distribution of lesions, the predominance of opacification in ground- glass, fine cross-linking and thickening of the peribronchovascular system, as well as the rarity $(<5 \%)$ of pleural effusions and lymphadenopathy in SARS-CoV-2 infection [15].

Evolution generally sees the initially pure frosted glass, transforming into an aspect of crazy paving due to the secondary appearance of intra lobular reticulations (peak around the 10th day) possibly associated with areas of condensation, more extensive in the forms severe [1]. According to the French Society of Radiology, there are very likely to be carriers of the virus and contagious without clinical or scanographic aspect [13].

\section{Lung Ultrasound for Pulmonary COVID-19}

Lung ultrasound allowed the identification of $\mathrm{nCoV}-19$ infection at bed-side. Moreover, lung ultrasound can have several other advantages, such as reduced health worker exposition to infected patients, repeatability during followup, low-costs and easier application in low-resource settings [16].

A recent study reports the echographic aspects in 20 patients with pneumonia due to COVID-19, its aspects range from interstitial syndrome with multiple B lines to alveolar syndrome via all intermediates of severity (aspect in snowstorm with lines B coalescing, sub-pleural alveolar syndrome...) [17]. However, the French Society of Radiology has limited the place of ultrasound for patients with COVID-19, because this technique, unlike CT, does not allow the distinction between viral pneumonia, bacterial or interstitial edema of cardiogenic origin which can occur in case of viral myocarditis, as the technique requires prolonged contact with the patient, risk of contamination for the operator, which is not the case with the scanner, in this sense, it recommends its interest only in resuscitation, in non-transportable patients, to identify complications of ventilation (pneumothorax) and to evaluate pleural effusions [18].

\section{CT features in pediatric patients with SARS-CoV-2 infection}

According to a recent study, the clinical signs of children with COVID-19 are similar to those of adults, such as fever and cough. Some children have diarrhea and a runny nose, but the overall symptoms are relatively mild [19].

Chest CT findings in children were similar to those in adults, and most of them were mild cases. The typical manifestations were unilateral or bilateral subpleural ground-glass opacities, and consolidations with surrounding halo sign [20].

\section{CONCLUSION}

In conclusion, it seems that the role of the radiologist in this epidemic lies first in the detection of early abnormalities of viral pneumonia.

The extension of CT lesions must be specified because it is correlated with the clinical severity of the disease. Progress towards an Acute Respiratory Distress Syndrome (ARDS) should in particular be mentioned. Finally, the radiologist must know how to suggest a possible bacterial superinfection or differential diagnoses.

\section{ACKNOWLEDGEMENT}

The authors want to acknowledge the editorial office of the journal all the anonymous reviewers.

Declaration of interest: The authors report no conflicts of interest.

Financial Disclosure: No financial support was received. 


\section{REFERENCES}

1. Dai W-C, et al. CT imaging and differential diagnosis of COVID-19. Canadian Association of Radiologists Journal, 2020; 71(2): 195-200. doi: $10.1177 / 0846537120913033$.

2. Heller L, Mota CR, Greco DB. COVID-19 faecal-oral transmission: Are we asking the right questions? Science of the Total Environment 2020: 138919. doi: 10.1016/j.scitotenv.2020.138919

3. Zhang D, et al. Potential spreading risks and disinfection challenges of medical wastewater by the presence of Severe Acute Respiratory Syndrome Coronavirus 2 (SARS-CoV-2) viral RNA in septic tanks of fangcang hospital. medRxiv, 2020.04.28.20083832. doi: $10.1101 / 2020.04 .28 .20083832$.

4. Rodriguez-Morales AJ, et al. Clinical, laboratory and imaging features of COVID-19: A systematic review and meta-analysis. Travel medicine and infectious disease, 2020: 101623. doi: 10.1016/j.tmaid.2020.101623.

5. Huang $\mathrm{C}$, et al. Clinical features of patients infected with 2019 novel coronavirus in Wuhan, China. The lancet, 2020. 395(10223): 497-506. doi: 10.1016/S01406736(20)30183-5.

6. Wan Y, et al. Receptor recognition by the novel coronavirus from Wuhan: an analysis based on decadelong structural studies of SARS coronavirus. Journal of virology 2020; 94(7): e00127-20. doi: 10.1128/JVI.0012720.

7. Baba MA, Bouchriti Y, Achbani A, Kharbach A, Sine H, Naciri A. Risk of COVID-19 for patients with cancer: A narrative overview. European journal of medical and educational technologies, 2020; 13(3): em2008. doi: $10.30935 /$ ejmets/8257.

8. Salehi S, et al. Coronavirus disease 2019 (COVID-19): a systematic review of imaging findings in 919 patients American Journal of Roentgenology, 2020: 1-7. doi: 10.2214/AJR.20.23034.

9. Soldati G, et al. Proposal for international standardization of the use of lung ultrasound for COVID-19 patients; a simple, quantitative, reproducible method. Journal of Ultrasound in Medicine First published: 30 March 2020. doi: 10.1002/jum.15285.
10. Garg M, et al. Spectrum of imaging findings in pulmonary infections. Part 1: Bacterial and viral. Polish journal of radiology 2019; 84: e205. doi: 10.5114/pjr.2019.85812

11. Fang Y, et al. Sensitivity of chest CT for COVID-19: comparison to RT-PCR. Radiology 2020: 200432. doi: 10.1148/radiol.2020200432.

12. Pan F, et al. Time course of lung changes on chest CT during recovery from 2019 novel coronavirus (COVID19) pneumonia. Radiology 2020: 200370. doi: 10.1148/radiol.2020200370.

13. Revel, Lederlin, Brillet, Khalil. La société d'imagerie thoracique, Epidémie de Covid-19: Point Sur L'Imagerie. Societe Francaise de Radiologie 2020. Available at: https://cutt.ly/6yRW0pe

14. Ng M-Y, et al. Imaging profile of the COVID-19 infection: radiologic findings and literature review. Radiology: Cardiothoracic Imaging, 2020; 2(1): e200034. doi: 10.1148/ryct.2020200034.

15. Bai HX, et al. Performance of radiologists in differentiating COVID-19 from viral pneumonia on chest CT. Radiology, 2020: 200823. doi: 10.1148/radiol.2020200823.

16. Buonsenso D, et al. novel coronavirus disease-19 pnemoniae: a case report and potential applications during COVID-19 outbreak. European review for medical and pharmacological sciences, 2020; 24: 2776 80. doi: 10.26355/eurrev_202003_20549.

17. Ruan Q, et al. Clinical predictors of mortality due to COVID-19 based on an analysis of data of 150 patients from Wuhan, China. Intensive care medicine, 2020: 1-3. doi: 10.1007/s00134-020-05991-x.

18. Revel, Lederlin, Brillet, Khalil. La société d'imagerie thoracique - sit, COVID-19: Mise au point sur la place de l'échographie thoracique. Societe Francaise de Radiologie, 2020. Available at: https://cutt.ly/IyREjat.

19. Xia W, et al. Clinical and CT features in pediatric patients with COVID-19 infection: Different points from adults. Pediatric pulmonology, 2020; 55(5): 116974. doi: $10.1002 /$ ppul.24718.

20. Kanne JP. Chest CT findings in 2019 novel coronavirus (2019-nCoV) infections from Wuhan, China: key points for the radiologist. Radiological Society of North America,2020;295:16-7.doi:10.1148/radiol.2020200241. 